\title{
Une sémiotique à refaire?
}

\author{
Eric Landowski
}

Résumé: Le récit, c'est ce qui nous sert à donner un peu de signification et de valeur à la vie, en dépit de ce qu'elle peut avoir d'insignifiant par sa monotonie ou d'insensé par son aspect chaotique. Mais indépendamment des significations portées les récits, il nous arrive parfois, face à autrui, à une œuvre, à un paysage, d'être saisis par la présence d'un sens qui s'impose à notre intuition bien qu'il n'émane d'aucun discours constitué. Ce sens second, toute une littérature, de Proust à Musil, de Svevo à Woolf, s'est employée à le dire en inventant les formes d'écriture de ce qu'on peut appeler le discours de l'expérience. Distinct du vécu, ce discours a le pouvoir d'en capter le mouvement en restituant la dynamique du rapport aux choses, à autrui, à soi-même sur laquelle se fonde l'expérience du sens dans son émergence en acte. Pour construire une sémiotique qui permette d'en rendre compte, ni la grammaire narrative standard ni ses prolongements relatifs aux passions et à la tensivité ne fournissent les instruments nécessaires. Il convient donc de compléter l'appareil théorique dont nous disposons. C'est ce qui justifie le réexamen critique des notions de narrativité et de discursivité auquel procède cet article, ainsi que la proposition finale d'un modèle tenant compte de la diversité des régimes possibles de production du sens dans l'interaction.

Mots-clefs: ajustement; esthésie; expérience; interaction; narrativité; sensible

Abstract: Re-making Semiotics? - In daily life, we are often searching for signs at the surface of the world, as if it were a text. But it also happens that in the absence of any particular signs which would convey some codified significations we feel the presence of elements that immediately make sense. Not only music, images, faces, but any object may affect us this way: we sensitively grasp what they mean, rather than we cognitively read them as signs. Semiotics should develop tools in order to account for both these modes of significance. The present essay reconsiders critically, with this scope, the basic semiotic notions of narrativity and discursivity, and proposes a model articulating different regimes of meaning at play in human interaction.

Keywords: adjustment; aesthesic; experience; interaction; narrativity; sensitive

\section{Du vécu à l'énoncé, et retour}

Le temps est-il venu pour une sémiotique existentielle? C'est ce que nous envisagions il y a quelques années en conclusion de Passions sans nom (LANDOWSKI, 2004, p. 293-305) 
sans avoir eu connaissance des travaux entrepris autour de cette même idée, bien que dans une optique différente, par Eero Tarasti (2000; 2009). Qu'il n’y ait là qu'une coïncidence ou davantage, une telle orientation ne ferait que renouer avec l'ambition qui animait au départ le courant sémiotique dont nous nous réclamons. Telle est en tout cas notre conviction, quitte peut-être à surprendre ceux qui voient la sémiotique « greimassienne » de l'extérieur et à heurter quelques habitudes de pensée chères à certains de ceux qui la pratiquent.

Aux yeux des sémioticiens de stricte obédience structurale, pour tenir un discours sensé à propos du sens il n'existe en effet qu'une façon de procéder: c'est d'analyser les productions manifestes à travers lesquelles le sens se donne à appréhender, à commencer par ces « manifestations » signifiantes par excellence que sont les textes. Autant cette option paraît offrir de garanties de scientificité, autant il serait aventureux et même, pour les plus orthodoxes, oiseux de s'interroger sur les implications existentielles de l'expérience toute "subjective » que nous vivons lorsque nous éprouvons sur un mode impressif la présence autour de nous d'un monde qui tantôt semble faire sens, tantôt non, sans que nous puissions dire au juste pourquoi ni comment. "Faire sens », "présence », "impressif », «éprouver », « expérience », " vécu »... I'emploi d'un tel vocabulaire fait aujourd'hui encore l'objet de fortes résistances malgré les efforts déployés déjà de longue date par quelques-uns en vue de l'intégrer au métalangage sémiotique ${ }^{1}$. Cela sans doute parce que ce vocabulaire ne paraît pas d'emblée compatible avec les procédures de l'analyse textuelle. Et il est vrai que si Greimas et quelques autres n'avaient pas dans un premier temps pris le parti d'analyser les textes (qu'ils soient de type folkloriques, mythologiques ou littéraires) abstraction faite des impressions esthétiques ou des réactions morales que leur lecture peut susciter, aucun des modèles opératoires dont nous disposons à présent n'aurait probablement pu être construit.

Mais cela n'a pas été sans contrepartie. La focalisation sur le texte a eu pour effet d'éloigner les tenants de l'approche structurale des autres courants de réflexion sémiotique, notamment de celui issu de Peirce, qui vise, on le sait, la construction d'une théorie de la signification envisagée dans sa plus grande généralité. Pourtant, chez Greimas aussi le ressort initial de la réflexion était une interrogation sur le sens en général. Chez lui, au départ, ce n'était pas seulement de la signification des textes qu'il devait être question mais du "sens des activités humaines », du "sens de I'histoire » ou tout simplement, selon une expression qui lui était familière, du « sens de la vie » (GREIMAS, 1966, p. 5; GREIMAS; COURTÉS, 1979, p. 245). Dans Sémantique structurale, son premier livre, cherchant à refonder la sémantique pour en faire une théorie capable de dépasser les limites des approches lexicales, linguistiques ou logiques alors dominantes, il fait fi des précautions épistémologiques d'usage et se fixe comme cadre de réflexion l'horizon le plus large qu'on puisse concevoir: il part de «la situation de l'homme » et de ce qui en constitue le trait premier et décisif, à savoir le fait que pour chacun d'entre nous, habiter

1 Cf. notamment Geninasca (1997a); Greimas (1987); Landowski (in CALAME, 1988); Henault (1994); Parret (2001). 
le monde, c'est « du matin au soir et de l'âge prénatal à la mort » être « assailli par les significations qui [nous] sollicitent de partout»(GREIMAS, 1966, p. 8). Et a contrario, peut-on ajouter, serait-ce encore « vivre » qu'habiter un monde réduit à l'insignifiance ou à l'insensé? Autrement dit, c'est bien de notre condition existentielle, en tant qu'êtres sémiotiques, qu'il était alors question.

Mais en même temps, la "vocation scientifique » assignée au projet sémiotique dès les premières pages du même livre (conformément à son sous-titre, Recherche de méthode) commandait la plus grande vigilance contre le risque de verser dans un discours impressionniste ou psychologisant, ou de s'en tenir à une réflexion de type spéculatif qui n'aurait pu aboutir qu'à une philosophie du langage parmi d'autres. Pour se constituer comme théorie et comme méthode opératoire, la sémiotique structurale devait concevoir son objet, le sens, de manière positive, comme un objet de science. C'est ce qui a conduit à passer d'une réflexion générale concernant le sens vécu à une problématique du sens manifesté - première réduction - et de là, seconde réduction, à une analytique du texte énoncé, en oubliant du même coup et pour longtemps « la vie ». La sémiotique est ainsi devenue, peut-être, une "science » — sans aucun doute une méthode — mais déconnectée de ce qui, pour une discipline vouée à la quête du sens, reste quoi qu'on dise la seule question qui vaille : mieux comprendre comment, dans quelles conditions, par quelles procédures notre présence au monde en vient à faire sens.

Ce que nous nous proposons est un retour à cette interrogation première car nous croyons que les deux termes de l'opposition implicitement admise - la science d'un côté, de l'autre, la vie — ne sont pas inconciliables mais qu'au contraire la dimension existentielle, vécue, de notre expérience du sens peut, à la fois comme thème de réflexion et comme matière à conceptualiser, faire l'objet d'une approche rigoureuse, en termes sémiotiquement articulés. Bien qu'une telle visée ait des connotations plus philosophiques que ce dont se préoccupe la majorité des sémioticiens, c'est en sémioticien et non en philosophe que nous la concevons et prétendons la justifier. Les démarches que cela appelle de notre part sur le plan conceptuel ne sont commandées par aucun impératif, philosophique ou autre, qui serait extérieur à la sémiotique mais s'imposent comme des exigences inséparables du métier de sémioticien tel que nous le concevons. D'abord, à quoi bon «faire de la sémiotique » - pourquoi s'interroger sur le sens — si cela ne devait aider à mieux se poser par rapport à soi-même la question du sens « de la vie », de sa propre vie? Et plus techniquement, second ressort de notre démarche, c'est de la pratique même des modèles sémiotiques, ainsi que de l'examen critique que cette pratique amène inévitablement à en faire, que découle à nos yeux la nécessité et la possibilité de rouvrir le champ de la réflexion et des analyses.

Il n'y a là ni désir de provocation ni la prétention d'invalider les principes de la discipline à l'intérieur de laquelle nous nous situons. Il s'agit au contraire d'en amplifier la portée, d'en revivifier la pratique et d'en enrichir les enjeux. Le renouvellement que nous 
proposons ne conduit donc nullement à négliger les résultats des recherches menées sur les formes de la textualité. Loin d'en contester l'utilité, nous y voyons un acquis indispensable qui, au prix d'un certain nombre d'amendements et de compléments, devrait précisément permettre aujourd'hui de reformuler la question existentielle originaire.

\section{State of the art}

Mais revenons au geste premier de Greimas consistant à se donner pour point de départ notre condition existentielle même, «la situation de l'homme » envisagée comme une totalité à l'intérieur de laquelle le sujet énonçant et en principe connaissant, le sémioticien, se trouve lui-même enclos, comme tout un chacun. C'est de là qu'il prétend fonder une science ! Véritable aporie car si l'univers sémantique s'identifie au monde de la vie à l'intérieur duquel nous sommes « définitivement enfermés»(GREIMAS, 1966, p. 117), et si par ailleurs une théorie sémantique ne peut se concevoir que comme une description d'ordre métalinguistique hiérarchiquement distincte de son langage-objet, on ne voit pas alors comment on pourrait traiter sémantiquement de l'univers englobant qu'on vient de se donner pour objet tout en s'y reconnaissant soi-même englobé. S'il nous inclut, nous ne pouvons pas l'appréhender du dehors ou « de plus haut » comme le prescrit pourtant le préfixe même de méta-langage. Mais c'est là que Greimas invente une solution de génie.

\section{II.1. Avatars et promesses d'une invention}

"Le mieux qu'on puisse faire », écrit-il, face à cette "situation » indépassable — au fait que nous appartenions à l'univers même du sens à analyser —, " c'est encore de prendre conscience de la vision du monde qui s'y trouve impliquée, à la fois comme signification et comme condition de cette signification ». En d'autres termes, puisque I'univers signifiant n'est pas objectivable de l'extérieur et à distance, la seule possibilité qui s'offre est de l'analyser « de l'intérieur » en faisant apparaître ce qui " s'y trouve impliqué »: une certaine "vision du monde».

Cette « vision » ne consiste pas en une projection de contenus qui donneraient au monde perçu une coloration déterminée, romantique ou apocalyptique par exemple. C'est $d^{\prime}$ une grille de lecture à caractère structurel qu'il s'agit, d'un principe d'organisation en fonction duquel le monde prend forme — se laisse découper et articuler — et par suite fait sens. Et la manière dont cette grille structure notre vision du monde et le fait apparaître devant nous sous la forme $d^{\prime}$ un " univers signifiant » consiste, dit Greimas, à nous le faire voir comme un "petit spectacle » indéfiniment susceptible de se reproduire, à des « millions d'exemplaires »(GREIMAS, 1966). Sur cette scène du monde qui devient ainsi langage (c'est-à-dire signifiant), ou mieux, discours (car l'univers signifiant est un univers en mouvement, faute de quoi ce ne serait pas un spectacle mais tout au plus un tableau), 
« le contenu des actions change tout le temps, les acteurs varient, mais l'énoncé-spectacle reste le même »(GREIMAS, 1966, p. 173).

A partir de là s'est édifié ce que la sémiotique structurale offre de plus original, à savoir le rabattement de la problématique de la signification sur une théorie de la narrativité. L'invariant auquel renvoie la vision structurante dont il est ici question — l'élément qui restera "le même » sous la surface d'énoncés-spectacles toujours différents — n'est autre en effet que la syntaxe interactantielle dont la grammaire narrative aura charge de dégager les unités - « un procès, quelques acteurs, une situation plus ou moins circonstanciée » (GREIMAS, 1966, p. 117) — et d'expliciter les régularités de fonctionnement. Moyennant I'observation de corpus textuels variés, cette grammaire sera systématisée durant les années 1970². Elle constitue le noyau de la théorie " greimassienne ». Diffusée sous le nom de modèle standard, elle reste aujourd'hui la base d'applications où se reconnaît, souvent à la lettre, l'enseignement prodigué voilà plus de trente ans.

Revers prévisible, ce qui avait été radicalement novateur s'est peu à peu figé, d'autant plus que l'efficacité du modèle, sur le plan opératoire, favorisait l'exercice routinier d'un "faire descriptif » strictement programmé qui ne pouvait laisser place à aucune interrogation sur les fondements de la théorie, sur ses présupposés idéologiques ou ses implications anthropologiques. Et l'idée de narrativité, qui en elle-même reste des plus prometteuses, allait bientôt se refermer dans les limites d'un schéma narratif pompeusement qualifié de " canonique » comme s'il fallait, à des fins pédagogiques, proclamer indépassable la syntaxe actantielle particulière qu'il met en œuvre, en dépit de ce que son auteur lui-même y voyait de contingent, de partiel et de provisoire, donc de propre non pas à clore la réflexion mais à l'ouvrir et à la stimuler ${ }^{3}$. Pourtant, le travail de recherche proprement dit ne s'est pas arrêté là, en tout cas pas pour tout le monde, et pour Greimas certainement moins que pour quiconque, lui à qui il arrivait de dire, en privé, que la sémiotique était « à refaire »(GENINASCA, 1997b, p. 42).

Pour croire à la possibilité de la relancer en retrouvant un peu du tonus intellectuel des débuts et, en même temps, nous convaincre qu'il était possible de renouer le lien avec l' « existentiel », il nous a suffi de relire Greimas et de porter un regard critique sur notre propre pratique. Ce qui nous est alors apparu, c'est que si la plupart des objections émises de l'extérieur contre les travaux des " greimassiens » sont justes, elles sont aussi dépassables. Et dépassables de l'intérieur de la théorie (GENINASCA, 1997b ; BUCHER, 1997 ; LANDOWSKI, 1997b; KERSYTE, 2009). C'est la raison pour laquelle nous nous employons depuis des années à dépasser les blocages qui ont contribué à étouffer les potentialités de la discipline, à l'empêcher de se développer comme démarche réflexive et analytique en prise sur la vie.

2 Cf. principalement Greimas; Courtes (1979).

3 Cf. Greimas; Courtés (1979, p. 247), entrée « Narratif (schéma) ». 


\section{II.2. Ce que nous faisons des textes et ce qu'ils font de nous}

Le premier obstacle à surmonter n'était autre que la confusion qui s'était établie à propos de l'objet même de la sémiotique. Greimas y était pour quelque chose, lui qui, au fil d'une conférence, lança un jour cette formule, source d'un malentendu qui allait se révéler persistant: " Hors du texte, point de salut! »S'adressant à un public de spécialistes de la littérature, il s'agissait pour lui, assez trivialement, d'inviter ses auditeurs à être logiques avec eux-mêmes, à travailler sur la " littérature » — sur les textes considérés comme littéraires — au lieu de spéculer, comme il était alors d’usage, sur les paramètres d'ordre social, affectif et autres ayant pu intervenir au stade de la genèse des œuvres.

Mais ce n'est pas ce qui a été entendu. La formule a été interprétée comme un oukase traçant une frontière entre deux zones: d'une part les textes - les discours verbaux, de préférence écrits - , qu'on s'est mis à considérer comme le fief du sémioticien, et d'autre part, tout autour, zone soi-disant décrétée interdite, le " contexte », c'est-à-dire rien moins que la société, l'histoire, le réel, la vie ! C'était tout simplement confondre objet empirique et objet de connaissance. Car si les textes sont des objets empiriques qui de toute évidence intéressent les sémioticiens, l'objet de connaissance, lui, n'est pas le texte: c'est le sens. Or le sens, à la différence du trésor caché dans la caverne d'Ali Baba, n'est pas une chose qu'on puisse trouver toute constituée en se donnant seulement la peine de pénétrer à l'intérieur de la " clôture du texte », comme s'il y avait un jour été déposé et attendait qu'on vienne en prendre possession.

A partir du moment où on admet au contraire qu'aucun objet — texte ou autre ne contient de cette manière sa signification mais que tout peut s'en voir attribuer une à condition qu'un sujet la construise, on doit admettre aussi que le sens qui sera attaché, en particulier, à un texte dépend constitutivement à la fois de l'intertexte pris en compte par celui qui effectue ladite construction, et du " contexte » à l'intérieur duquel elle est effectuée. Ou plutôt, pour employer une terminologie plus adéquate, un discours énoncé (verbal ou non) prend son sens en fonction de la manière dont les partenaires de la communication construisent le sens de la situation où prend place l'acte d'énonciation qui les met en rapport. Les composantes d'une telle situation sont sémiotiquement analysables ${ }^{4}$. Il s'agit entre autres choses de l'image que chacun des interlocuteurs se fait de son partenaire, combinaison variable de compétences modales et de rôles thématiques définissant leurs statuts actantiel et actoriel respectifs en tant que sujets interagissants. Une des stratégies communes à la plus grande variété de discours consiste, comme on sait, à installer dans l'énoncé des simulacres figurant les participants à la relation énonciative en vue d'orienter l'activité interprétative du récepteur en le conduisant vers une position de lecture déterminée, celle par exemple d'un objecteur ou d'un confident, ou encore, comme souvent dans la publicité, d'un voyeur ${ }^{5}$.

4 Cf. Landowski (1989), "Quelques conditions sémiotiques de l'interaction »; Landowski (1997a), " Pour une sémiotique des situations ».

5 Cf. Greimas ; Landowski, (1979) ; Landowski, (2007b). 
La tâche d'un sémioticien ne peut donc pas se limiter à statuer sur ce que les textes pourraient signifier à raison de leurs seules structures « immanentes ». Dans la perspective d'une recherche motivée par un minimum d'intérêt pour les enjeux existentiels ou, plus modestement, psychologiques, sociaux, politiques, des pratiques quotidiennes du sens, plus pertinent que I'acharnement à hypostasier le texte en tant que forme pure nous semble I'objectif consistant à chercher à rendre compte de ce que les objets de sens, lus en situation, font de nous qui les lisons. Autrement dit, à partir du moment où on considère que ce qui importe, c'est ce qui se passe dans le vécu de la lecture conçue comme opération donnant sens à des objets quelconques, ce sont les conditions de cette lecture qui s'imposent comme principal objet sémiotique à décrire ${ }^{6}$.

Mais les éléments modaux, actantiels et thématiques au moyen desquels se construisent les simulacres discursifs des partenaires de l'interlocution, de leur statut, de leur compétence et de leurs relations ne sont pas les seuls à entrer en jeu dans la construction d'une situation en tant que configuration signifiante. Interviennent aussi des variables qui, relevant davantage du sensible que de l'intelligible au sens étroit, c'est-à-dire du cognitif, mobilisent notre aptitude, presque innée, à saisir des effets de sens ressortant de dispositifs d'ordre plastique ou rythmique plutôt que notre compétence, tant bien que mal acquise, à reconnaître les significations conventionnellement investies dans des unités figuratives. En un mot, en deçà du cognitif, le sens — l'intelligibilité du monde — procède aussi de l'esthésique.

Et c'est là qu'on rencontre un autre point de blocage.

\section{Narration ou expérience?}

Sous prétexte que la dimension sensible des objets de sens ne paraît pas réductible à un jeu d'oppositions catégoriques aisément dénommables et que, de plus, on voit mal comment elle pourrait s'articuler aux schématisations syntaxiques que propose la grammaire narrative « canonique », les adeptes de la sémiotique standard jugent en général prudent de l'ignorer: par opposition à la positivité des articulations syntactico-sémantiques permettant de rendre compte de l'action et des passions dans le cadre d'analyses textuelles en bonne et due forme, l'expérience sensible relèverait de l'ineffable.

Il y a là un étrange paradoxe car rien ne saurait être plus substantiel, plus concret, plus incarné (moins ineffable) et en même temps moins flou, plus exactement structuré, que les agencements de qualités plastiques et rythmiques — en un mot, esthésiques — par lesquels font sens des manifestations de type sémiotiquement aussi divers qu'un morceau de musique ou un mouvement de foule (accompagné dans la rue ou vu à la télévision), que les expressions changeantes d'un visage ou simplement la manière dont quelqu'un se tient devant autrui au fil d'une conversation. Ce qui se donne alors à voir, à entendre,

6 Cf. "Le regard impliqué », (LANDOWSKI, 2004). 
à sentir, n'a certes pas une signification du genre de celle que nous attribuons à un mot, à une phrase, à un texte. Ce n'en est pas moins d'une forme de communication qu'il s'agit, face à face ou corps à corps, et qui nous affecte. Le fait qu'elle s'instaure en deçà des mots n'implique pas que ses effets de sens n'offrent aucune régularité. Par suite, rien n'autorise à postuler que ses principes de fonctionnement devraient échapper à toute forme de connaissance autre qu'intuitive.

En tout cas, ce qui intervient à ce niveau influe si fortement sur le sens que nous attribuons à notre présence les uns aux autres — qui est lui-même le premier élément du " sens de la vie » - qu'on ne voit pas comment on pourrait, en tant que sémioticien, se résigner à n'en tenir aucun compte. Le fait qu'il y ait là, en outre, une dimension essentielle de l'expérience esthétique est une évidence. Mais en dehors même du rapport aux œuvres d'art, cette dimension intervient pratiquement partout, y compris dans des domaines dont on pourrait souhaiter qu'ils ne soient pas soumis aux fluctuations de la sensibilité. Notamment dans la politique, domaine que nous prendrons comme exemple pour justifier ce point.

\section{III.1. Entre histoire et présence}

Comme les politiciens parlent et écrivent beaucoup, on a pris depuis longtemps le pli d'analyser leurs discours. On s'est d'abord attaché à relever les contenus idéologiques, doctrinaux, programmatiques qui s'y expriment, puis à décrire les procédures d'argumentation, de persuasion ou de manipulation qui y sont à l'œuvre. Et plus récemment, constatant que ce genre de discours s'adresse autant à l'affectivité qu'à l'intellect, on a cherché à rendre compte aussi des émotions et des passions qu'ils sont censés produire ou entretenir. Mais le « passionnel », dimension sémiotiquement réductible à une syntaxe modale, présuppose lui-même une autre dimension, plus profonde, celle du sensible, qui n'est, elle, sémiotiquement analysable qu'en termes d'esthésie.

Aujourd'hui, du fait de leurs constantes apparitions dans les médias, les hommes politiques les plus en vue nous sont devenus si familiers que la manière dont nous les jugeons ne diffère guère de celle dont nous considérons nos proches. Si nous estimons certains d'entre eux, si d'autres nous exaspèrent, cela ne dépend pas uniquement des positions qu'ils adoptent sur telle ou telle question importante à nos yeux. Cela tient tout autant aux effets de sens d'ordre esthésique qui se dégagent de la tenue, de la complexion, du rythme, du ton de voix de chacune de ces personnalités, en un mot, de leur hexis propre tel qu'il se manifeste à travers le moindre geste, traduisant une manière spécifique $d^{\prime}$ être au monde. La manière dont nous les écoutons, dont nous comprenons et jugeons leurs arguments, et jusqu'à notre degré de disponibilité à réagir favorablement aux états passionnels qu'ils cherchent à nous faire partager, tout cela dépend de ce que nous éprouvons face à leur personne même, telle que nous la « sentons » d'emblée. 
Sans doute n'y a-t-il là rien de radicalement nouveau et pourtant cette inflexion en faveur du sensible affecte de plus en plus le statut, les formes, le lieu même du « politique » (LANDOWSKI, 2008). Autrefois, pour le citoyen moyen, la politique se situait dans un espace un peu abstrait, construit par les discours d'un nombre limité de détenteurs de la parole autorisée, journalistes et politiciens en premier lieu, qui se chargeaient de donner un sens à l'histoire, d'expliquer le présent et de tracer pour l'avenir des interprétations plausibles, telles que la vie collective paraisse se dérouler comme une sorte de grand récit intelligible. Une autre manière de vivre la politique tend aujourd'hui à s'affirmer: on a cessé de l'envisager uniquement comme un niveau de réalité objectivable dans des énoncés ayant pour forme type les petites épopées ou les grandes utopies que les orateurs politiques se sont toujours fait un devoir de nous raconter; au lieu de cela, elle tend à devenir un espace de rapports intersubjectifs vécus dans l'ici-maintenant à la faveur d'actes d'énonciation qui, par médias interposés, impliquent la co-présence sensible de ceux qui parlent et de ceux qui les regardent et les entendent parler. Cette relation est à la fois ténue - elle se vit dans l'instant - et profonde car elle procède du rapport, quasi intime, que chacun entretient ou croit entretenir à l'égard de ces hommes et de ces femmes en chair et en os qui, pour rallier nos suffrages, se montrent à qui mieux mieux devant nous. On veut pour cela des politiciens qui ne se cachent pas derrière un supposé savoir, qui ne se tiennent pas à distance ou au-dessus de nous, bref, qui ne nous "racontent pas d'histoires », ou en tout cas ne s'en tiennent pas à cela. Ce qu'on attend d'eux, c'est qu'ils se risquent à un rapport in praesencia censé permettre de sentir si, oui ou non, ils nous " comprennent ».

La politique, domaine, à ce qu'on dit, du calcul et de la froide raison, tend ainsi à devenir un des plans sur lesquels la dimension existentielle de notre rapport à autrui affleure à la surface, se traduisant sous forme de gestes d'adhésion ou de rejet à fleur de peau. On en a eu un exemple probant en France à l'occasion d'un débat crucial entre les deux candidats du second tour de l'élection présidentielle de 2007. Ce soir-là, beaucoup d'électeurs qui hésitaient à choisir leur camp — ceux que les politologues qualifient de "volatiles» — ne se sont sans doute ralliés à I'un ou l'autre candidat qu'en fonction de la sympathie ou de l'antipathie que le contraste entre les qualités esthésiques du mode de présence de chacun d'eux vis-à-vis de son adversaire aussi bien qu'à l'égard des deux journalistes présents, et finalement face à la caméra, c'est-à-dire aux électeurs, pouvait faire immédiatement ressentir pour l'un ou pour l'autre. Pour l'un et contre l'autre serait à vrai dire plus exact.

Ce déplacement du politique, on peut le cerner conceptuellement en disant que si naguère, pour tout le monde ou presque, la politique avait de la signification, aujourd'hui où, d'après les enquêtes d'opinion, elle n'en a plus pour grand monde, elle fait sens selon un régime sémiotique tout différent: autant, vue comme narration, elle a perdu, cognitivement, de sa crédibilité, autant, vécue esthésiquement comme l'une des formes de l'expérience, elle retrouve sa capacité de mobilisation. 


\section{III.2. Le vécu et son discours}

Mais « la politique » n'est qu'une construction sémiotique parmi d'autres et les deux régimes de signifiance que nous venons de distinguer à son propos — l'expérience, la narration - ont une portée beaucoup plus générale.

Les narrations, c'est ce qui nous sert à donner un peu de signification et de valeur à la vie en dépit de ce qu'elle peut avoir d'insignifiant par sa monotonie ou d'insensé par son aspect imprévisible et chaotique. Mais indépendamment des significations portées par ce qui se raconte, il nous arrive parfois — face à autrui, face à une œuvre, face à un paysage — de nous sentir saisis par la présence $d^{\prime}$ un sens qui, bien que par hypothèse il n'émane d'aucun discours constitué, d'aucun récit en forme, s'impose immédiatement à notre intuition. Ce sens second (ou peut-être en réalité premier), la narration ne le raconte pas et ne peut pas le « raconter ». Pour le raconter, il lui faudrait qu'il soit rédutible à l'ordre des significations objectivées, textualisées, lisibles, " en papier», comme disait Greimas. Et par nature, ce genre de significations ne peut refléter que très imparfaitement les modulations du sens vécu dans l'expérience même de notre rapport aux gens ou aux choses.

Cependant, le fait que l'expérience, ainsi entendue — celle du sens —, ne soit pas racontable $n^{\prime}$ implique pas qu'elle soit indicible. Toute une littérature s'emploie au contraire à la dire. De Proust à Sarraute, de Kafka à Musil, de Sterne à Woolf, de Leopardi à Svevo, de Dostoïevski à Tsvetaïeva, une pléiade d'auteurs que rapproche le fait d'avoir projeté sur le monde un regard en quelque sorte phénoménologique avant la lettre ont su inventer des formes d'écriture constitutives de ce qu'on peut appeler le discours de l'expérience (LANDOWSKI, 2007a). Ce discours est certes distinct du vécu de l'expérience mais il a le pouvoir d'en capter le mouvement et de restituer discursivement la dynamique du rapport aux choses, à autrui, à soi-même sur laquelle se fonde l'expérience du sens dans son émergence en acte. Au point que devant ce type de textes il nous semble, à nous lecteurs, devenir partie prenante à la naissance du sens. Qui plus est, entre ce mode de traitement de l'expérience du sens sur le plan de l'écriture littéraire et son traitement par des voies scientifiques, la frontière est à peine discernable. Entre la démarche de Proust et celle de Merleau-Ponty, où la frontière passe-t-elle au juste? On ne voit pas davantage de rupture entre le Sartre de La Nausée et celui de la partie finale de L'être et le néant consacrée à l'analyse de l'expérience immédiate du "gluant », du " glissant », etc., analyses qui débouchent sur le projet d'une «psychanalyse existentielle».

Maintenant, pour construire une sémiotique existentielle à même de traiter de ce sens vécu, ni la grammaire narrative standard ni ses prolongements relatifs aux passions et à la tensivité ne fournissent les instruments nécessaires ${ }^{7}$. La sémiotique des passions ne fait qu'étendre à la syntaxe des états ( $d$ 'âme) la grammaire modale précédemment appliquée au « faire » des actants de la narration ; la sémiotique dite tensive fournit un

7 Cf. Greimas; Fontanille (1991); Fontanille; Zilberberg (1998). 
formalisme utile pour analyser les variations d'intensité de phénomènes quelconques mais ne nous dit rien sur les déterminants qualitatifs des effets de sens saisis par les sujets ${ }^{8}$. Le fait que dans ces conditions nous nous trouvions relativement démunis pour rendre compte de l'expérience est-il une raison suffisante pour ignorer tout ce qui échappe aux grilles d'analyse construites pour décrire la narration? Pour notre part, loin de tirer de la limitation de nos acquis théoriques et méthodologiques la conclusion que la question existentielle n'est pas pertinente ou que le statut du sens appréhendé dans l'expérience ne peut être que d'ordre extra-sémiotique, nous croyons que ce qui fait problème, c'est l'incomplétude des modèles dont nous disposons. Nous avons donc cherché à les compléter et à les rendre plus puissants.

\section{L'optique socio-sémiotique}

Passer d'une problématique du texte à une problématique des situations, puis de l'expérience, c'est étendre le champ de pertinence par rapport à la pratique sémiotique dominante. Cette extension correspond en même temps à un approfondissement, en ce sens qu'elle conduit de ce qui est le plus extérieur au sujet — le texte, dans son objectivité $d^{\prime}$ « énoncé énoncé » — à ce qui lui est le plus propre, le plus « intérieur » — l'expérience du sens telle qu'il la vit —, en passant par un niveau intermédiaire, la situation, comme configuration signifiante construite intersubjectivement par les co-participants de l'interaction.

Pour effectuer ce parcours, un corps de concepts nouveaux, entre autres ceux d'union, de contagion, d'ajustement, d'assentiment, d'accomplissement a dû être mis en place. Ils ont fait l'objet de définitions que nous ne reprendrons pas ici en détail ${ }^{9}$. Notre objectif se situe sur un plan plus global: nous voudrions montrer comment la théorie élargie à laquelle nous sommes abouti nous rapproche de la vie sans pour autant obliger à renoncer à la rigueur qu'exige une visée «scientifique ». Ce grand mot ne sert bien entendu qu'à enjoliver une démarche plus modeste qui peut se résumer ainsi: réaménagement des deux composantes de base — narrative et figurative — sur lesquelles se fonde la théorie sémiotique classique du discours, et renouvellement de la manière de les articuler l'une à l'autre. C'est ce que nous allons préciser dans ce qui suit.

\section{IV.1. Les logiques complémentaires de la jonction et de l'union}

A la base de la grammaire narrative dite standard se trouve l'hypothèse implicite selon laquelle toutes les fluctuations affectant notre condition matérielle et morale de sujets dépendent d'opérations de " jonction » qui tantôt nous mettent en possession des « objets de valeur » que nous sommes censés viser (conjonction), tantôt nous en privent (disjonction). Un tel modèle se justifie par rapport à un espace d'interaction conçu comme clos -

8 Concernant la justification de ces réserves, cf. Landowski (2004, p. 44-49) ; Landowski (2005, p. 94-96).

9 Cf. Landowski (2004); Landowski (2005). 
typiquement, celui du conte —, à l'intérieur duquel ce qu'un protagoniste perd, un autre doit nécessairement le recevoir en partage. Et il a l'avantage de se prêter aisément à une certaine formalisation. Mais il a l'inconvénient d'être idéologiquement très marqué. Une grammaire de l'intersubjectivité conçue comme entièrement médiatisée par la circulation des objets et débouchant sur des parcours de vie dont le sens ne peut tenir qu'à des rapports de possession traduit une vision extrêmement étroite, purement économique, des rapports humains. De l'avoir le plus concret au savoir le plus abstrait, tout s'y monnaie sous la forme de valeurs les unes thésaurisables ou consommables, les autres modales, qui ont vocation à transiter entre possesseurs à la manière de marchandises toujours en attente de quelque nouvel acquéreur (LANDOWSKI, 2004, p. 73-76).

La redécouverte des années 1990, liée à la parution du dernier livre de Greimas, De I'Imperfection, en même temps qu'à la relecture des phénoménologues français de I'après-guerre, c'est qu'il existe aussi, en tant que positivités sémiotiquement analysables, des interactions indépendantes de tout transfert d'objets entre sujets. Pour reprendre une expression de Sartre, indépendamment des rapports de possession qu'ils entretiennent vis-à-vis de ce qu'ils considèrent comme étant de l'ordre de l'avoir, les sujets vivent aussi, entre eux et par rapport à leur environnement, certains "liens d'être » (SARTRE, 1947, p. 325). Ou bien, selon une terminologie empruntée à Merleau-Ponty, avant de se décomposer en unités discrètes offertes à notre prise ou à notre convoitise, le monde nous est présent en tant que totalité faisant sens: c'est notre être au monde qui, en tant que tel, im-médiatement (sans passer par la médiation d'objets socialement catégorisés et valorisés), fait qu'il y a, ou qu'il peut y avoir du sens dans nos rapports à l'autre et, en général, au réel qui nous entoure (MERLEAU-PONTY, 1945).

En termes sémiotiques, cela revient à dire qu'à côté d'un régime de signification articulé autour de l'idée de jonction, on doit faire droit aussi à un autre régime de sens, fondé sur la coprésence sensible des actants les uns aux autres. Dans ce cadre, les objets ne sont plus réductibles à des grandeurs interchangeables dont la valeur s'apprécie sur la base de critères d'ordre fonctionnel fixés en référence à des programmes d'action prédéfinis. Appréhendés en tant que réalités matérielles, ils font sens à raison des qualités sensibles que peuvent y découvrir des sujets dotés de quelque chose d'essentiel qui leur manquait sous le régime narratif précédent: un corps. Eux qui n'étaient au mieux qu'intelligents, capables de connaître, de juger, de décider, d'évaluer à distance et du dehors, ils deviennent au surplus sensibles, sensoriellement réceptifs aux qualités esthésiques des êtres avec lesquels ils entrent en relation. Et dès ce moment — dès qu'un actant est à même de sentir esthésiquement l'autre qui lui fait face, de saisir sa manière propre d'être au monde — , la voie est ouverte pour une forme de rapports qui ne relèvent plus de la jonction mais de ce que nous appelons la logique de l'union. Ce rapport n'annulle pas les identités. Au contraire, il tend à les exalter en les faisant entrer en contact et interagir en fonction de leur sensibilité réciproque. L'union n'est donc pas la fusion; elle n'est d'ailleurs pas un état. C'est un mode d'interaction 
(et du même coup, un mode de construction de sens) dans le cadre duquel les parties en viennent — dans le meilleur des cas - à constituer ensemble, dynamiquement et par ajustement mutuel, une entité complexe nouvelle, une totalité inédite en laquelle chacune trouve une forme de son propre accomplissement ${ }^{10}$.

La complémentarité entre ces deux logiques, celle de la jonction et celle de l'union, permet d'intégrer I'ensemble des composantes de la compétence sémiotique - non seulement modale mais aussi esthésique — des sujets analysés. Qui plus est, cette dualité de régimes a vocation à s'appliquer aussi aux méta-sujets que nous sommes en tant que sémioticiens et analystes. Il y va de la possibilité même d'une sémiotique existentielle. Car une telle sémiotique ne peut se construire que dans un rapport dialectique avec ce qu'elle analyse: à la fois en union (selon un régime de relation sensible) et en position d'extériorité objectivante (selon le vocabulaire du type jonctif ou, aujourd'hui, tensif), quitte à ce que dans la pratique les tâches correspondantes soient provisoirement réparties entre des acteurs-chercheurs distincts. Pour notre part en tout cas, nous assumons I'intention de connaître autrement, en nous " unissant » dans toute la mesure du possible à l'autre. Quels que puissent être la forme et le statut de cet « autre» — interlocuteur, texte, œuvre ou élément du monde naturel —, il s'agit de le comprendre en l'approchant comme un sujet tenu pour sensible lui aussi, tout en sachant que d'autres chercheurs, soucieux d'en modéliser le fonctionnement en tant qu'objet, sauront s'en distancier méthodologiquement autant que nécessaire.

\section{IV.2. Régimes de sens dans l'interaction}

Une fois dépassées les limites du modèle jonctif, de nouvelles possibilités s'ouvrent pour une théorie générale de la construction et de l'appréhension du sens dans l'interaction. Commençons à ce propos par quelques généralités.

Quel que soit le projet à réaliser, la démarche à entreprendre, le problème à résoudre ou l'affaire à mener à bien, c'est un fait empiriquement constatable que chacun, dans la vie quotidienne, est enclin (en fonction de sa culture ou de quelque idiosyncrasie personnelle) à privilégier un modus operandi déterminé, un certain style d'action, une " stratégie » de préférence à telle ou telle autre. Beaucoup, ne se sentant en confiance que dans un environnement ordonné et maîtrisé, rêveraient de pouvoir programmer le comportement des gens aussi bien que le cours des choses de façon à s'assurer jusque dans le plus menu détail le contrôle du déroulement de la moindre opération dans laquelle ils se trouvent impliqués. D'autres, ne voyant partout que machinations et complots, pensent ne pouvoir arriver à leurs fins qu'en manipulant eux-mêmes, de manière aussi contournée que de besoin, ceux avec qui ils ont à traiter. D’autres encore préférent se fier à leur intuition, au flair, à leur capacité de sentir sur le moment les tenants et aboutissants d'une situation

10 Cf. "Jonction versus Union ", "Formes de I'accomplissement », (LANDOWSKI, 2004); " Le régime de l'ajustement », (LANDOWSKI, 2005). 
ou les dispositions de ceux auxquels ils ont affaire, prêts à s'y ajuster et à en tirer parti en saisissant l'occasion « aux cheveux ». D'autres enfin, écartant toute idée de plan, de calcul ou de syntonie avec autrui, croient plus sûr de s'en remettre à leur bonne étoile et se contentent de se croiser les doigts en attendant quelque accident, heureux ou malheureux, que la providence aura décidé pour eux et auquel ils donnent par avance leur assentiment.

Autant de manières d'être qui, tout en correspondant chacune à une façon de concevoir le sens de la vie, se traduisent dans des styles de conduite différenciés sur le plan des interactions avec les objets, avec autrui, avec soi-même. Ces variantes relèveraient du seul donné psychologique et nous n'aurions rien de plus à faire que de les constater si les régimes de sens et d'interaction auxquels elles renvoient ne s'articulaient les uns aux autres en fonction de principes structurels qui ne doivent rien à la psychologie mais se révèlent sémiotiquement analysables. Pourtant, parmi ces configurations qui, intuitivement, nous sont si familières, il se trouve que la sémiotique narrative n'en avait jusqu'à présent reconnu et thématisé que deux: I'« opération », ou action programmée sur les choses, et surtout la " manipulation », entre sujets. En reprenant les définitions classiques de ces deux régimes, il n'était pas difficile de faire apparaître les bases sur lesquelles ils reposent respectivement. Le premier est fondé sur un principe général de régularité; manifesté par l'immuabilité des « rôles thématiques » assignés aux protagonistes de l'action, c'est ce principe qui garantit (en principe) l'efficacité de nos interventions sur le monde environnant. Le second procède d'un principe d'intentionnalité dont la mise en œuvre suppose la reconnaissance réciproque des partenaires de l'interaction en tant qu'actants sujets dotés de « compétences modales » (du type vouloir, croire, etc.) sans cesse changeantes. C'est ainsi que la grammaire narrative a mis à I'honneur la figure du manipulateur et, plus accessoirement, celle du programmateur.

En revanche, ni le sujet confiant dans sa capacité de sentir in vivo les potentialités $d^{\prime}$ une situation, de tourner à son avantage la " propension des choses ${ }^{11}$ ou des gens, de saisir et d'exploiter le kairos — baptisons-le faute de mieux l'opportuniste —, ni le fataliste décidé à s'en remettre au hasard, ne trouvaient de place dans ce cadre. L'observation de l'interaction, et d'abord l'expérience que nous en avons obligeaient pourtant à les prendre aussi en considération. Pour analyser tant soit peu exhaustivement les pratiques effectives, il était par conséquent nécessaire d'ouvrir l'éventail des formes de la narrativité. D'où l'introduction, à côté des deux régimes « standards » mentionnés à l'instant (qui, en perdant leur monopole, ne perdent rien de leur pertinence) de deux régimes complémentaires fondés respectivement sur un principe de sensibilité et sur un principe d'aléa : le régime de I'« ajustement » (à l'autre) et celui de I'« assentiment » (aux décrets du sort, aux " accidents » de la vie) (LANDOWSKI, 2005, p. 72).

11 Expression empruntée à Jullien (1996). 


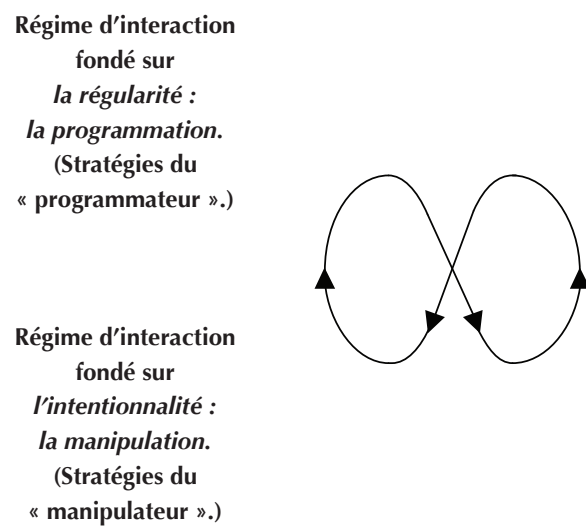

\author{
Régime d'interaction \\ fondé sur \\ I'aléa : \\ I'assentiment. \\ (Stratégies du \\ " fataliste ».) \\ Régime d'interaction \\ fondé sur \\ la sensibilité : \\ I'ajustement. \\ (Stratégies de $\mathrm{I}^{\prime}$ \\ " opportuniste ».)
}

Formant système et ayant vocation à se combiner entre elles, les quatre formules auxquelles nous aboutissons permettent de rendre compte de la variété et du caractère le plus souvent composite des pratiques interactionnelles observables sur les terrains les plus divers, y compris celui de la construction de l'objet de connaissance dans nos disciplines à visée scientifique ${ }^{12}$. Cependant, pour reformuler la question du sens « de la vie », et pour que ces réflexions débouchent sur la production d'instruments d'analyse, il ne suffit pas de repenser de cette manière les formes du " petit spectacle » que nous nous jouons pour constituer l'univers ambiant en monde signifiant, d'en dégager les principes implicites et de repousser les frontières de la narrativité. Le statut, les formes et le rôle de la figurativité, et plus encore la manière de concevoir son articulation avec la composante narrative, tout cela était aussi à réexaminer.

\section{Formes de l'être au monde}

Selon les circonstances, en fonction des contextes, du type d'objets qui se présentent et de la nature des rapports que nous cherchons à établir avec ce qui nous environne, nous oscillons, dans la vie même, entre deux manières très différentes de regarder le monde et de le faire signifier. Dans le cadre d'une problématique sémiotique de portée générale, I'une et l'autre doit être prise en considération.

\section{V.1. La lecture et la saisie}

Le plus souvent, nous regardons le monde comme s'il s'agissait d'une surface couverte de signes que nous avons appris à lire. La compréhension de ce à quoi nous sommes confrontés passe alors par le déchiffrement de formes manifestes qui, verbales ou non, constituent l'équivalent d'autant de textes supposés «vouloir dire » quelque chose ou,

12 Cf. Landowski (2006). 
selon la formule adoptée plus haut, " avoir de la signification ». Mais il arrive aussi que nous ne regardions plus, ou pas encore, le monde comme un réseau de signes à décrypter. Devant nous, il n'y a alors rien à déchiffrer, rien à lire, aucun texte. Et paradoxalement, il y a tout de même déjà, ou encore, du sens et de la valeur. C'est qu'à défaut de chercher partout, un peu obsessionnellement, les marques de discours intelligibles qui nous seraient adressés (intentionnellement ou non), nous osons quelquefois prendre le plaisir, et en même temps peut-être le risque, de lever nos préventions et de nous laisser imprégner sans réserve par les qualités sensibles inhérentes aux êtres et aux choses mêmes dont la présence nous environne. Au régime de la lecture du monde fait alors place celui de la saisie d'un monde vécu comme " faisant sens».

Il est vrai que nous pouvons souvent avoir le sentiment d'une concordance entre ces deux modalités du regard ou, plus généralement, entre ces régimes de signifiance. Ainsi, dans une conversation, tant que le ton de notre interlocuteur paraît correspondre au contenu de qu'il est en train de nous dire, nous n'avons aucun raison de dissocier la saisie de la lecture — l'esthésique du cognitif — puisque précisément les effets de sens que ses inflexions de voix nous permettent de capter sur le mode impressif se trouvent validés par leur conformité avec ce qui nous apparaît comme la signification articulée et intentionnelle de ses propos. La distinction n'en reste pas moins pertinente dans son principe, et utile dans la pratique dès qu'il cesse d'y avoir concordance. Mais surtout, elle vaut sur un autre plan, d'ordre métasémiotique, où se pose la question des implications « existentielles» qu'ouvre l'alternative entre ces régimes.

D'un côté, pour se faire lecteur et regarder le monde à la manière d'un texte, il faut que le sujet se détache de ce qu'il voit, l'objective, l'observe comme une réalité en ellemême signifiante, interprétable, potentiellement intelligible même si elle ne dévoile pas d'emblée sa signification. Vu selon cette perspective, un texte ou tout objet considéré comme tel est un objet autonome, tenu à distance et servant de support à des significations supposées déjà constituées, qu'il s'agit d’y découvrir. Si énigmatique puisse-t-il sembler à première vue, un tel texte est par définition présumé déchiffrable. Il suffit d'en trouver la clef. Toute lecture équivaut dans ces conditions à un décodage et, à ce titre, relève essentiellement d'une herméneutique, ou même d'une sémiologie lorsque les règles de codification sont suffisamment stabilisées et formalisées (par exemple grâce à quelque système « semi-symbolique ${ }^{13}$ ).

Par opposition, un procès indéfiniment ouvert (et proprement sémiotique) préside à l'émergence du sens dans l'expérience que constitue la saisie. Cet effet d'ouverture tient à ce qu'ici le sujet s'implique directement dans son rapport à l'objet: c'est dans le moment même où ce rapport se noue et le met à l'épreuve par la manière dont il s'articule que le sujet vit sa propre présence à l'objet comme faisant sens. Il en résulte qu'à la différence de la «signification », qui n'apparaît qu'au terme d'un travail de déchiffrement, le "sens »

13 Cf. Floch, (1986, p. 26-27 et passim). 
constitue, lui, un point de départ: saisi dans l'instant, c'est lui et non plus le texte qui fait énigme pour le sujet. C'est lui qu'il va devoir s'efforcer de comprendre, quitte, le cas échéant, à le recatégoriser dans les termes de quelque schéma de signification — de quelque narration - plus ou moins convenu. Ce sens que le sujet vient de saisir, quel sens - ou quelle signification — lui attribuer? L'énigme du texte-objet, la question de sa signification, était par nature, en principe, soluble par la lecture; en revanche, la question du sens - du sens du « sens vécu » dans l'expérience — n'a pas de solution de ce genre puisque le sens qui naît alors est celui de la relation même qui se noue hic et nunc entre le sujet et son objet: pour cette raison, l'énigme qu'il pose à celui qui en fait l'expérience est sans fin ${ }^{14}$.

En opposant la lecture des significations (qui relèvent de la narration) à la saisie des qualités sensibles (qui font sens dans l'expérience), nous ne voulons pas suggérer que le premier de ces régimes ne concernerait que nos rapports aux textes (en tant qu'objets lisibles par nature) ou plus généralement au verbal (conçu comme I'intelligible par excellence), par opposition au second, qui aurait alors trait spécifiquement au non verbal (du coup assimilé quant à lui au sensible). Chacun de ces régimes se définit par la nature du regard qu'il implique sur le monde et non par une classe particulière d'objets auquel il s'appliquerait exclusivement. Cela revient à dire que pour un sujet qui se place sous le régime de la lecture, n'importe quel objet peut tenir lieu de texte, autrement dit être regardé comme porteur de significations; et qu'inversement, pour qui se place sous celui de la saisie, tout objet, y compris un texte, peut être regardé comme faisant sens au-delà de ce qu'il signifie en termes linguistiques ou narratifs. Mais alors, comment signifie-t-il donc? - Tout simplement (si on peut dire) sur le mode esthésique, ou, bien que ce terme risque de faire problème, "figuratif ».

\section{V.2. Formes de la figurativité}

Dans l'architecture de la théorie sémiotique, la « figurativité » se définit, comme on sait, par rapport à la « narrativité »: ce sont là deux concepts génériques qui renvoient, le premier, à la composante sémantique du discours, le second à sa composante syntaxique. En revanche, quand elle est utilisée comme outil pour l'analyse d'un discours particulier, la notion de figurativité prend un caractère plus technique. Le mot désigne alors un ensemble de formes discursives à valeur iconique censées représenter les objets du monde, ou indicielle, chargées d'en signaler inférentiellement la présence: c'est la figurativité stricto sensu, dite aussi de surface ( $b$ ci-dessous) Mais à cela s'ajoute une troisième acception du terme car à côté de la figurativité de surface il a fallu reconnaître une figurativité dite profonde $(c)$ dont les effets de sens, relevant de l'esthésie, tiennent quant à eux à I'organisation rythmique et plastique (par exemple chromatique) des éléments dont se compose la manifestation discursive:

14 A titre d'exemple de ces deux postures, analysées respectivement à partir d'un texte de Lévi-Strauss et d'une page de Proust, cf. Landowski (2004, p. 294-303). 


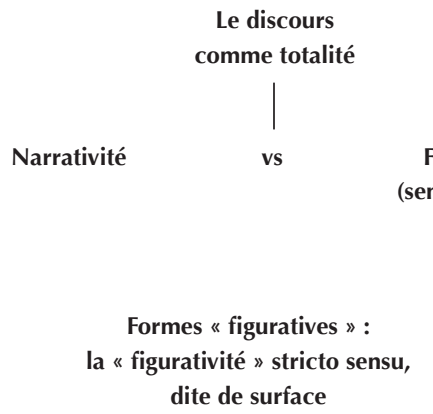

(b)

\section{Figurativité \\ sens générique)}

(a)

vS
Formes " plastiques " :
la " figurativité " lato sensu,
dite profonde

(c)

Pendant longtemps, aussi bien en sémiotique visuelle qu'en sémiotique littéraire, le traitement du « figuratif » a été ramené à la formule du type (b): on se bornait à reconnaître à la surface des discours certaines figures actorielles, spatiales ou temporelles plus ou moins strictement codifiées par l'usage, qu'on considérait comme chargées de donner leur « habillage discursif » aux dispositifs plus abstraits mis en forme par la syntaxe narrative. C'est à ce principe qu'obéit tout bon lecteur: attentif au moindre indice susceptible de " vouloir dire » quelque chose, il relie les uns aux autres les éléments de la manifestation discursive — décors, costumes, physionomies, gestes, etc. — et, à partir d'eux, cherche à reconstituer la structure actantielle et modale sous-jacente aux actions et aux passions dont le spectacle du monde, vu comme narration, tirera finalement sa signification.

Au contraire, envisagée selon la formule (c), la figure impose la présence du sens tel qu'il émane des articulations plastiques immanentes à l'objet sensoriellement perceptible. Greimas parle à ce propos d'une « langue autre », d'un " langage second » à l'aide duquel non seulement les œuvres — picturales, poétiques ou autres — mais aussi les objets du monde naturel nous « parlent autrement »: autrement qu'à travers le filtre des langues naturelles et des grilles culturelles que nous avons appris à leur appliquer pour les lire en y reconnaissant des « objets nommables» (GREIMAS, 1984, p. 12). On peut toutefois se demander si, plutôt que second, ce langage plastique ou, plus généralement, esthésique, qui ne désigne rien et pourtant nous parle par le jeu des qualités sensibles qu'il met en œuvre, n'est pas en réalité premier. $C^{\prime}$ est ce que Greimas suggère quand il observe que « la narration n'apparaît, dans toute sa figurativité débordante, que comme le "bruit" qu'il faut surmonter pour pouvoir dégager les principales articulations de l'objet »(GREIMAS, 1984, p. 24). Ce bruit de la narration, c'est celui de la figurativité de surface à laquelle s'attache la lecture. Par opposition, les articulations principales — principales non pas dans l'absolu mais parce qu'elles donnent accès à ce qu'on peut considérer comme le sens « profond » de l'objet, comme son sens « mythique » ou encore existentiel — sont celles qui organisent les formes plastiques constitutives de la figurativité profonde dont nous faisons l'expérience à travers les procès de la saisie ${ }^{15}$.

15 Sur l'idée d'une hiérarchie entre régimes de sens et sur le privilège qu'on peut accorder à la saisie par rapport à la lecture, cf. Lévi-Strauss (1955, p. 121) et notre commentaire in Landowski (2004, p. 180-182). 
Cela dit, un dernier pas reste encore à effectuer. II ne suffit pas en effet, bien que ce soit d'usage, de traiter un peu formellement de la figurativité dans un chapitre à part. Si on veut mesurer toute la portée du rôle qu'elle joue dans l'émergence du sens, il faut la mettre en relation avec les formes que recouvre le modèle présenté plus haut en ce qui concerne la narrativité, étant donné que ce sont là deux composantes indissociables de tout discours. Relativement à la composante figurative, nous venons de distinguer deux grands régimes de signifiance : la lecture et la saisie, la première définie comme décryptage des significations attachées au figuratif stricto sensu, la seconde comme procédure faisant émerger du sens à partir de la figurativité dite profonde (autrement dit, à partir de l'esthésique). Par ailleurs, nous avons construit un modèle narratif étendu interdéfinissant quatre régimes d'interaction (la programmation, la manipulation, I'ajustement, I'assentiment). II s'agit maintenant d'envisager les rapports entre ces deux dispositifs, notre postulat étant que des corrélations systématiques s'établissent entre régimes de signifiance et régimes d'interaction.

\section{V.3. Styles existentiels}

La première de ces corrélations consiste en ce que le décryptage des significations à travers la lecture du monde — de ses figures de surface — va de pair, sur le plan de I'interaction, avec des comportements relevant de l'un ou de l'autre des deux principes dont la théorie sémiotique standard fait le ressort grammatical de toute narration: soit du principe d'intentionnalité qui fonde la syntaxe de la manipulation, soit du principe de régularité que présuppose toute programmation, soit $d^{\prime}$ une combinaison des deux.

Cette corrélation, ce n'est pas nous qui la posons, inspiré par quelque vision a priori ; et elle n'est pas non plus le fruit du hasard. Elle trouve sa raison d'être dans le fait que le procès de la lecture est lui-même déjà, en réalité, de l'ordre de la programmation. Toute lecture n'a-t-elle pas en effet pour condition de possibilité la régularité du code qu'elle met en œuvre pour la reconnaissance des significations du texte ou, plus largement, du monde vu comme surface textuelle? Et lire, n'est-ce pas en outre, par nature, entrer dans le jeu de la persuasion et de l'interprétation, autrement dit de la manipulation? Dans les termes du Dictionnaire de Greimas et Courtés, cela revient à dire que toute lecture a au fond pour "programme d'usage » une programmation et pour " programme de base » une manipulation ${ }^{16}$. L'affinité que nous relevons entre ces régimes, entre une manière déterminée d'interpréter le monde et une manière correspondante d'y agir, est donc d'ordre proprement structural. Et on pourrait montrer qu'une relation du même ordre associe la saisie à l'autre paire de régimes d'interaction, à savoir l'ajustement et l'assentiment. Mais nous nous bornerons à deux illustrations.

Un parfait exemple de la première corrélation se trouve chez Stendhal, dans Le Rouge et le Noir, avec le personnage de Julien Sorel. Lire-programmer-manipuler,

16 Cf. Greimas ; Courtés (1979, p. 298), entrée « Programme narratif ». 
ou mieux, lire afin de pouvoir programmer ou manipuler, telle est la combinaison de modes opératoires qu'on le voit adopter en toutes circonstances, que ce soit pour analyser ses relations avec autrui, pour arrêter ses décisions ou d'une manière générale pour orienter ses comportements. Dans tous les domaines — mondain, amoureux, politique —, Julien est à la fois, sur le plan de la signifiance, un lecteur, et sur le plan de l'action un intrigant, programmé pour manipuler. Qui plus est, le programme de vie qui le guide est lui-même tiré d'une lecture (le Mémorial de Sainte-Hélène) en sorte que tout son parcours existentiel procède explicitement d'une narration première qui lui sert de norme: pour lui, la narration préfigure la vie et se substitue à l'expérience. Osons donc cette petite impertinence: en tout cela, dans cet acharnement à dominer le monde en lui appliquant coûte que coûte les procédures mêmes que formalisent les schémas canoniques des années 1970 et ceux, dits "pathémiques », qui en sont dérivés, Julien Sorel ne serait-il pas en somme une incarnation anticipée du parfait Sémioticien Standard? — Quoi qu'il en soit, de même que nous avons admis la possibilité d'un « discours de l'expérience » distinct du « vécu de l'expérience » mais ayant le pouvoir de s'élever à sa hauteur et de le prendre sémiotiquement en charge, on voit qu'il faut maintenant admettre au surplus que le discours de la narration a, lui aussi, un pouvoir par rapport au vécu: non plus le pouvoir de l'exalter en le recréant mais, à l'inverse, celui de l'encadrer par avance dans ses grilles, et par là de le réduire sinon de le rabaisser au rang d'un simple vécu de la narration.

Le cas diamétralement opposé nous est fourni par un personnage de Dostoïevski, M. Goliadkine, dans Le Double. Incapable de maîtriser les codes du milieu où il évolue, il ne parvient à dégager aucune signification des intrigues qui se trament autour de lui : $c^{\prime}$ est un langage qu'il ne sait pas « lire ». En revanche, à peine entre-t-il en contact avec autrui que prolifèrent à ses yeux, sur le mode de la saisie, une multitude d'effets de sens dramatiquement énigmatiques qui procèdent ou bien du principe de sensibilité ou bien du principe d'aléa. C'est dire que dans son rapport aux formes de la figurativité, $M$. Goliadkine est typiquement un non-lecteur; sensible jusqu'à l'excès aux qualités plastiques des manifestations auxquelles il a affaire, il ne perçoit son entourage que comme dans un rêve, ou plus exactement un cauchemar. Et dans l'interaction, c'est-à-dire en termes de narrativité, il est exactement le contraire d'un manipulateur programmé: il se comporte (à sa manière, c'est-à-dire avec une extrême maladresse) comme une sorte de danseur, en ce sens que toutes ses motions ne sont jamais que des réactions immédiates aux impulsions sensibles venues de l'autre, auquel il cherche à chaque instant à s'ajuster (sans y parvenir). A quoi s'ajoute le fait qu'en véritable fataliste, il est par avance intimement résigné aux coups du sort qu'il anticipe tout en en déniant, pour ainsi dire magiquement, l'imminence.

La symétrie entre les deux personnages est presque parfaite. Le héros de Stendhal est aussi réfractaire aux données esthésiques de l'expérience que celui de Dostoïevski est aveugle aux signes conventionnels de la narration. Si l'imperméabilité de Julien aux 
appels du sensible fait de lui (comme le narrateur le souligne à maintes reprises) un « sot », I'aveuglement de Goliadkine face aux significations les plus élémentaires du texte social fait de lui un idiot, un ridicule, un marginal et bientôt un psychopathe destiné à l'asile. Tandis que I'un, en observateur distant de son entourage, d'autant plus lucide qu'il est détaché aussi de lui-même, planifie froidement ses actions en anticipant les réactions d'autrui, l'autre, comme dans une succession d'hallucinations, se trouve ballotté par une suite d'accidents esthésiques imprévisibles dont l'irruption lui interdit toute stratégie efficace. Et finalement, de même qu'il faudra attendre les derniers chapitres du roman de Stendhal pour que Julien, une fois emprisonné, entrevoie ce qu'aurait pu être l'expérience du sens, de même, il faut que M. Goliadkine ait son double - un « M. Goliadkine numéro deux », manipulateur retors qu'on prendrait presque pour un autre Julien Sorel - pour que le régime « canonique » de la narration trouve aussi sa place dans le roman de Dostoïevski.

De telles connexions entre régimes de sens et régimes d'interaction impliquent qu'à un certain niveau de profondeur, on a affaire à des dispositifs de caractère plus général qui les subsument. Déterminant en même temps la manière préférentielle dont un sujet est porté à faire signifier les réalités qu'il côtoie et celle selon laquelle il tend à interagir avec ces mêmes réalités, de tels dispositifs traduisent la manière, proprement existentielle, d'être au monde à laquelle tient l'identité des personnages en question et sans doute aussi - avec toutes les variantes, nuances et complexifications imaginables — celle de chacun d'entre nous. En termes très généraux, alors que certains vivent la vie plutôt sur le mode de l'expérience, c'est-à-dire de la saisie et de l'ajustement-assentiment, d'autres semblent la concevoir, et même la vivre, plutôt comme une narration, le monde se présentant à leurs yeux comme un univers à la fois lisible parce que couvert de figures nommables et intelligibles, et maîtrisable, c'est-à-dire peuplé de partenaires ou d'adversaires manipulables et d'objets destinés par nature à se conformer à leurs programmes ${ }^{17}$.

\section{Conclusion}

Chacune des configurations sémiotiques qui nous ont occupé recouvre par conséquent à la fois, du point de vue de la théorie générale du sens, certains modes déterminés de traitement de la figurativité, et du point de vue de la théorie de l'action certaines formes précises de narrativité, le tout permettant de rendre compte de la différenciation des types $\mathrm{d}^{\prime}$ attitudes et de pratiques que chaque individu (ou, par extension, chaque culture) tend à privilégier face à la question du sens de la vie. C'est dire que traiter de l'expérience n'oblige en aucune façon - pas plus que décrypter la narration — à sortir du champ conceptuel où les notions de figurativité et de narrativité trouvent leur pertinence. Simplement, de même que la figurativité de surface, privilégiée par le discours de la narration, diffère

17 Sur la " philosophie " propre à chacune de ces " constellations » (terme que nous empruntons à G. Simmel), l'une vue comme sphère de l'aventure, I'autre de la prudence, cf. Landowski (2005, p. 77-79). 
de la figurativité profonde qu'on voit principalement à l'œuvre dans l'expérience, de même les modèles de narrativité de type programmatique et manipulatoire qui dominent la narration diffèrent de ceux, en forme d'ajustements et d'assentiments, qui articulent fondamentalement l'expérience. Une fois ces derniers reconnus, explicités, conceptualisés, le champ existentiel où ils interviennent s'ouvre à nos analyses.

Mais ces distinctions ont aussi une portée plus générale. Compte tenu de tout ce qui précède, la « narration », c'est au fond l'ensemble des discours qui fixent l'identité, la régularité, la nécessité et la rationalité de ce qui est. II s'agit d'un discours de l'assertion: discours qui connaît et qui, face à l'expérience incertaine du réel, permet de fixer des significations reconnaissables, lisibles parce que supposées par avance installées dans la forme manifeste de ce qui est. A l'inverse, l'expérience est interrogation sur le sens des effets de sens qui surgissent de ce qui advient au sujet, soit du dehors sous forme de figures esthésiques qui l'impressionnent, le touchent, l'affectent, soit du dedans de lui-même sous la forme d'impulsions relevant de la dynamique du corps propre et peut-être aussi — surtout si on opte pour inclure le rêve dans le champ de l'expérience-qui-fait-sens — de figures fantasmées traduisant les pulsions de " l'inconscient». Ces fluctuations, le sujet peut se laisser porter par elles ou au contraire s'efforcer de les maîtriser en tentant de les réduire à des schèmes empruntés à l'univers de la narration. Mais il peut aussi chercher à les dire par la production d'un discours de l'expérience à même de ressaisir réflexivement et de restituer, pour soi et le cas échéant pour autrui, ce qui a d'abord fait sens dans le vécu de l'expérience, c'est-à-dire dans une saisie initiale. Car si, on l'a noté, l'expérience n'est pas racontable, elle n'en est pas moins, en principe, dicible moyennant l'invention de formes d'expression, et en particulier d'écriture adéquates, littéraires bien sûr, ou cinématographiques, mais aussi phénoménologiques, et même, pourquoi pas, un jour, sémiotiques?

On le voit, il n'est pas nécessaire de verser dans le mysticisme pour parler de l'expérience, que ce soit comme vécu ou comme discours, et pour chercher à la mettre en parallèle avec le discours, et même le vécu, de la narration. Textualisées ou non, ce sont là autant de configurations aussi positives les unes que les autres. Et par suite, si la dimension existentielle de notre rapport au sens en constitue l'enjeu, on peut en entreprendre l'analyse sans déchoir de la sémiotique.

Eric Landowski est directeur de recherche associé au Cevipof (Paris, CNRS-Sciences Po), directeur de la revue Actes Sémiotiques, co-directeur du Centre de recherche socio-sémiotique de Sao Paulo et professeur invité à I'université de Vilnius. Ses recherches concernent la théorie de l'interaction, la sémiotique des pratiques quotidiennes et la dimension sensible du vécu. Principales publications: 
La société réfléchie (Seuil, 1989), Présences de l'autre (PUF, 1997), Passions sans nom (PUF, 2004), Les interactions risquées (Pulim, 2006).

\section{Références}

BUCHER, G., "De la perfection de la théorie à l'imperfection des lettres ", in AAVV, Lire Greimas, Limoges, Pulim, 1997.

FLOCH, J.-M., Les formes de l'empreinte, Périgueux, Fanlac, 1986.

FONTANILLE, J. et Cl. Zilberberg, Tension et signification, Liège, Mardaga, 1998.

GENINASCA, J., " Le regard esthétique », Actes Sémiotiques, VI, 58, 1984 (rééd. in La parole littéraire, Paris, P.U.F., 1997).

— «Et maintenant », in AAVV, Lire Greimas, Limoges, Pulim, 1997.

GREIMAS, A.J., Sémantique structurale, Paris, Larousse, 1966.

— «Sémiotique figurative et sémiotique plastique », Actes Sémiotiques, VI, 60, 1984.

— De l'Imperfection, Périgueux, Fanlac, 1987.

— et J. Courtés, Sémiotique. Dictionnaire raisonné de la théorie du langage, Paris, Hachette, 1979.

— et E. Landowski, Introduction à l'analyse du discours en sciences sociales, Paris, Hachette, 1979.

— et J. Fontanille, Sémiotique des passions, Paris, Seuil, 1991.

HENAULT, A., Le pouvoir comme passion, Paris, Presses Universitaires de France, 1994.

JULLIEN, Fr., Traité de l'efficacité, Paris, Grasset, 1996.

KERSYTE, N., "La sémiotique d'A.J. Greimas entre logocentrisme et pensée phénoménologique ", Nouveaux Actes Sémiotiques (http://epublications.unilim.fr/revues/as/index.php), 112, 2009.

LANDOWSKI, E., " La lettre comme acte de présence » in CALAME, Cl., (éd.), La lettre, Fribourg, Editions Universitaires de Fribourg, 1988 (rééd. in LANDOWSKI, 1997a).

— La société réfléchie. Essais de socio-sémiotique I, Paris, Seuil, 1989.

— Présences de l'autre. Essais de socio-sémiotique II, Paris, PUF, 1997.

— « Le sémioticien et son double», in AAVV Lire Greimas, Limoges, Pulim, 1997.

- Passions sans nom. Essais de socio-sémiotique III, Paris, PUF, 2004.

— Les interactions risquées, Limoges, Pulim, 2005.

— «L'épreuve de l'autre », Sign Systems Studies, 34, 2, 2006.

— «Unità del senso, pluralità di regimi », in AAVV, Narrazione ed esperienza, Rome, Meltemi, 2007.

— «L triangle publicitaire», Semiotica, 163, 2007.

— « La politique spectacle revisitée : manipuler par contagion », Versus, 107, 2008.

LEVI-STRAUSS, Cl., Tristes Tropiques, Paris, Plon, 1955. 
MERLEAU-PONTY, M., Phénoménologie de la perception, Paris, Gallimard, 1945.

PARRET, H., «Présences », Nouveaux Actes Sémiotiques, 76, 2001.

SARTRE, J.-P., L'être et le néant, Paris, Gallimard, 1947.

TARASTI, E., Existencial Semiotics, Bloomington, Indiana University Press, 2000 ;

— Fondements de la sémiotique existentielle, Paris, L’Harmattan, 2009.

Artigo recebido em agosto e aprovado em setembro de 2013. 